\title{
Exhaled volatile organic compounds as markers for medication use in asthma
}

\author{
Paul Brinkman ${ }^{1}$, Waqar M. Ahmed ${ }^{2}$, Cristina Gómez $\mathbb{1}^{3,4}$, Hugo H. Knobel ${ }^{5}$, \\ Hans Weda (10 ${ }^{6}$, Teunis J. Vink ${ }^{6}$, Tamara M. Nijsen ${ }^{6}$, Craig E. Wheelock (1) ${ }^{4}$, \\ Sven-Erik Dahlen ${ }^{3}$, Paolo Montuschi ${ }^{7}$, Richard G. Knowles ${ }^{8}$, \\ Susanne J. Vijverberg ${ }^{1}$, Anke H. Maitland-van der Zee ${ }^{1}$, Peter J. Sterk ${ }^{1}$ and \\ Stephen J. Fowler (10) ${ }^{2,9}$ on behalf of the U-BIOPRED Study Group ${ }^{10}$
}

Affiliations: ${ }^{1}$ Dept of Respiratory Medicine, Amsterdam UMC, University of Amsterdam, Amsterdam, The Netherlands. ${ }^{2}$ Division of Infection, Immunity and Respiratory Medicine, School of Biological Sciences, Faculty of Biology, Medicine and Health, The University of Manchester, Manchester, UK. ${ }^{3}$ Institute of Environmental Medicine and the Centre for Allergy Research, Karolinska Institutet, Stockholm, Sweden. ${ }^{4}$ Division of Physiological Chemistry 2, Dept of Medical Biochemistry and Biophysics, Karolinska Institutet, Stockholm, Sweden. ${ }^{5}$ Philips Signify, Eindhoven, The Netherlands. ${ }^{6}$ Philips Research, Eindhoven, The Netherlands. ${ }^{7}$ Dept of Pharmacology, Catholic University of the Sacred Heart, Fondazione Policlinico Universitario Agostino Gemelli IRCCS, Rome, Italy. ${ }^{8}$ Knowles Consulting. Stevenage Bioscience Catalyst, Stevenage, UK. ${ }^{9}$ Manchester Academic Health Science Centre, Manchester University Hospitals NHS Foundation Trust, Manchester, UK. ${ }^{10}$ The list of U-BIOPRED Study Group contributors can be found in the supplementary material.

Correspondence: Paul Brinkman, Dept of Respiratory Medicine, F5-259, Amsterdam UMC, University of Amsterdam, Meibergdreef 9, 1105 AZ Amsterdam, The Netherlands. E-mail: p.brinkmandamsterdamumc.nl

@ERSpublications

Exhaled volatile organic compounds can be linked to urinary traces of salbutamol and oral corticosteroids. This suggests that breathomics qualifies for development into a point-of-care tool for monitoring asthma drug level changes. http://bit.ly/2Hu4TOf

Cite this article as: Brinkman P, Ahmed WM, Gómez C, et al. Exhaled volatile organic compounds as markers for medication use in asthma. Eur Respir J 2020; 55: 1900544 [https://doi.org/10.1183/ 13993003.00544-2019].

\section{ABSTRACT}

Introduction: Asthma is a heterogeneous condition, characterised by chronic inflammation of the airways, typically managed with inhaled bronchodilators and corticosteroids. In the case of uncontrolled asthma, oral corticosteroids (OCSs) are often prescribed. Good adherence and inhalation technique are associated with improved outcomes; however, it is difficult to monitor appropriate drug intake and effectiveness in individual patients. Exhaled breath contains thousands of volatile organic compounds (VOCs) that reflect changes in the body's chemistry and may be useful for monitoring drug pharmacokinetics/pharmacodynamics. We aimed to investigate the association of exhaled VOCs in severe asthma patients from the U-BIOPRED cohort (by gas chromatography coupled with time-of-flight mass spectrometry) with urinary levels of salbutamol and OCSs (by liquid chromatography coupled with high-resolution mass spectrometry).

Methods: Samples were collected at baseline and after 12-18 months of follow-up. Statistical analysis was based on univariate and multivariate modelling, followed by area under the receiver operating characteristic curve (AUC) calculation. Results were verified through longitudinal replication and independent validation.

Results: Data were available for 78 patients (baseline $\mathrm{n}=48$, replication $\mathrm{n}=30$ and validation $\mathrm{n}=30$ ). Baseline AUC values were 82.1\% (95\% CI 70.4-93.9\%) for salbutamol and 78.8\% (95\% CI 65.8-91.8\%) for OCS. These outcomes could be adequately replicated and validated. Additional regression analysis between qualified exhaled VOCs and urinary concentrations of salbutamol and prednisone showed statistically significant correlations $(\mathrm{p}<0.01)$.

Conclusion: We have linked exhaled VOCs to urinary detection of salbutamol and OCSs. This merits further development of breathomics into a point-of-care tool for therapeutic drug monitoring. 


\section{Introduction}

Asthma is a heterogeneous disease, characterised by variable airflow obstruction and chronic inflammation of the airways. Control of asthma in general is managed with inhaled bronchodilator therapy and inhaled corticosteroids (ICSs). Short courses of oral corticosteroids (OCSs) are often prescribed in uncontrolled asthma and acute exacerbations, while maintenance therapy with OCSs can be required in patients with severe disease [1]. Good adherence and adequate inhalation technique are associated with improved outcomes; however, it is difficult for clinicians to monitor appropriate medication intake and drug effectiveness in individual patients [2,3].

In addition to pulmonologists, the World Anti-Doping Agency and the International Olympic Committee are interested in monitoring asthma treatments because of ergogenic effects. Although most bronchodilators and steroids are prohibited in-competition and require a Therapeutic Use Exemption, use of both drugs is still under debate. An important element of this discussion is the significant variability among individuals in the metabolism of the drugs [4-6]. Therefore, techniques suitable for monitoring these drugs in both athletes and patients are needed.

Exhaled breath contains thousands of volatile organic compounds (VOCs) that reflect changes in the body's chemical metabolism, since they are byproducts of metabolic and pathological processes at the cellular and tissue level. Metabolomic analysis of expired VOCs (breathomics) is commonly based on analytical methods such as gas chromatography (GC) and mass spectrometry (MS) and/or cross-reactive gas sensor electronic nose (eNose) technology, and is suggested to be used for: 1) diagnosis of disease, 2) disease phenotyping, 3) prediction of exacerbations and 4) prediction of treatment response [7-11]. Furthermore, it has been shown that some exhaled VOCs exhibit characteristic behaviour shortly after the onset of exercise [12]

Since the lungs are in direct contact with the blood circulation, analysis of exhaled volatiles may also be employed to monitor uptake and distribution of drugs [13, 14]. As breath sampling is noninvasive, (virtually) inexhaustible and, for the purposes of the Human Tissue Act, constitutes a "nonbiological material", it potentially is a preferable source to detect biological responses and metabolism kinetics of drugs than blood and urine. Despite all this, the analysis of exhaled VOCs for pharmacokinetics purposes has thus far received limited attention $[13,14]$.

Given that breathomics has real potential in the field of pharmacokinetics, we hypothesised that there is an association between markers of recent drug use in urine, as current gold standard, and exhaled VOCs. We therefore aimed to detect asthma medications in urine of severe asthma patients from the U-BIOPRED (Unbiased BIOmarkers in PREDiction of respiratory disease outcomes) project [15] by liquid chromatography (LC)-MS and to link those to exhaled VOCs or breath profiles measured by GC-MS.

\section{Methods}

Participants

In this study, data from an unselected subset of adult subjects (aged $\geqslant 18$ years) participating in the pan-European U-BIOPRED cohort study were included [15]. All participants were diagnosed with severe asthma according to Innovative Medicine Initiative criteria [16]. In short, patients were prescribed high-dose ICS (at least $1000 \mu \mathrm{g}$ per day fluticasone propionate or equivalent) plus at least one other controller medication and were defined uncontrolled according to Global Initiative for Asthma guidelines [1] and/or had at least two severe exacerbations in the preceding year and/or required prescription of daily OCS (maintenance) to achieve asthma control. Potential participants were excluded if they had an exacerbation in the previous month prior to enrolment. The study was approved by all local medical ethics committees and all patients gave written informed consent. The original study was registered at ClinicalTrials.gov with identifier number NCT01976767.

\section{Design}

The U-BIOPRED study in adults comprised three visits as reported in detail elsewhere [15]. At the first visit participants were screened for eligibility to participate according to the inclusion and exclusion criteria. Of relevance to the present study, several measurements were performed. In rank of order according to study protocol, the assessments during the baseline visit (second visit) and at the 1218-month follow-up visit (third visit) were: urine sampling and completion of questionnaires, followed by pre- and post-bronchodilator spirometry testing. Next, exhaled nitric oxide fraction was measured and exhaled breath collected, which was within 60 min from urine sampling. Finally, blood was drawn and allergy tests were carried out, all as outlined previously [15]. All baseline measurements were defined as the training set, while the 12-18-month follow-up samples were split into a replication set (revisits) and a validation set (previously unsampled patients). 


\section{Methods of measurement}

\section{Exhaled breath collection and analysis}

Exhaled breath was collected at eight participating sites using a previously described method [9, 10]. In short, patients breathed for $5 \mathrm{~min}$ at tidal volume through a two-way nonrebreathing valve and an organic compound filter (A2; North Safety, Middelburg, The Netherlands). Next, the subjects exhaled a single vital capacity volume into a $10 \mathrm{~L}$ Tedlar bag (SKC, Eighty Four, PA, USA). The exhaled VOCs were then trapped on sorbent tubes containing Tenax (Tenax GR SS $6 \mathrm{~mm} \times 7$ inch; Gerstel, Mülheim an der Ruhr, Germany) by pulling the air through the Tenax tube using a peristaltic pump at a flow rate of $250 \mathrm{~mL} \cdot \mathrm{min}^{-1}$. Such storage of VOCs preserves the exhaled marker signal [17].

At the Philips Research laboratory (Eindhoven, The Netherlands), Tenax tubes were thermally desorbed using helium as carrier gas. The sample was transmitted to a packed liner, heated to $300^{\circ} \mathrm{C}$ for 3 min and subsequently transferred to a Tenax TA cold trap $\left(\right.$ at $-150^{\circ} \mathrm{C}$ ), which was heated after 2 min to $280^{\circ} \mathrm{C}$ at $20^{\circ} \mathrm{C} \cdot \mathrm{s}^{-1}$ and splitless injected onto the chromatographic column. Compounds were separated using capillary GC with helium as a carrier gas at $1.2 \mathrm{~mL} \cdot \mathrm{min}^{-1}$ (7890 N GC; Agilent, Santa Clara, CA, USA) on a VF1-MS column $(30 \mathrm{~m} \times 0.25 \mathrm{~mm}$, film thickness $1 \mu \mathrm{m}, 100 \%$ dimethylpolysiloxane; Varian Chrompack, Middelburg, The Netherlands). The temperature of the gas chromatograph was adjusted in three steps: $40^{\circ} \mathrm{C}$ for $5 \mathrm{~min}$, increased until $300^{\circ} \mathrm{C}$ at $10^{\circ} \mathrm{C} \cdot \mathrm{min}^{-1}$ and finally held isothermally for $5 \mathrm{~min}$. A time-of-flight mass spectrometer (Pegasus 4D; LECO, Sint Joseph, MI, USA), in electron ionisation mode at $70 \mathrm{eV}$, was used for the detection of product ions (ranging from 29 to $400 \mathrm{Da}$ ).

\section{Urine collection and analysis}

Before the visit, patients were asked not to take food and drinks that were diuretic. The urine sample was split into five $8 \mathrm{~mL}$ tubes (Sarstedt, Nümbrecht, Germany). The capped tubes were immediately stored in the vertical position in a freezer $\left(-20^{\circ} \mathrm{C}\right)$.

All urine samples were analysed at the Dept of Medical Biochemistry and Biophysics of the Karolinska Institutet (Stockholm, Sweden). The sample preparation for determining fragments or metabolites of salbutamol and corticosteroids was performed on a robotic liquid-handling platform (Microlab STAR; Hamilton Robotics, Bonaduz, Switzerland). Corticosteroids and salbutamol were prepared from an aliquot of urine fortified with internal standards and subsequently hydrolysed using $\beta$-glucuronidase (Escherichia coli). Purification was performed using mixed-mode solid-phase extraction in 96-well plates. The analysis of the extract was performed with reversed-phase LC coupled to high-resolution MS (LC-HRMS) (Thermo Q-Exactive; Thermo Fisher Scientific, Waltham, MA, USA). Acquisition of raw LC-HRMS data was performed in full scan mode at a resolution of 35000 with polarity switching.

As a next step, two summarising, dichotomous variables were established regarding the observation of salbutamol and corticosteroid residues in a sample. Presence of corticosteroids was determined based on the remains of prednisone and/or prednisolone and/or methylprednisolone and/or 16 $\alpha$-hydroxyprednisolone and/or 20ß-dihydro-prednisolone [18, 19]. These two outcomes were used to split the patients into groups (salbutamol detected: yes/no and corticosteroids detected: yes/no) for statistical analysis. The limit of detection for all these compounds was $1 \mathrm{ng} \cdot \mathrm{mL}^{-1}$ [20], with an expected terminal half-life of 4-6 h for salbutamol and 2-4 h for prednisolone (https://farmacotherapeutischkompas.nl).

\section{Lung function}

Spirometry was executed before and $10 \mathrm{~min}$ after intake of $400 \mu \mathrm{g}$ of salbutamol via a spacer according to European Respiratory Society (ERS) recommendations using daily calibrated lung function equipment [21].

\section{Blood}

Blood eosinophils and neutrophils percentages were obtained from standard complete blood counts.

\section{Allergic status}

Allergy testing was performed using total and specific serum IgE and the skin prick test (SPT) to a panel of common aeroallergens. Atopy was defined as the presence of sensitisation on the SPT (wheal $\geqslant 3 \mathrm{~mm}$ ) or serum-specific $\operatorname{IgE}\left(\geqslant 0.35 \mathrm{kU} \cdot \mathrm{L}^{-1}\right)$.

\section{Exhaled nitric oxide fraction}

Fractional exhaled lower respiratory nitric oxide was measured with a portable device (NIOX Mino System; Aerocrine, Solna, Sweden) at a constant flow rate of $50 \mathrm{~mL} \cdot \mathrm{s}^{-1}$, according to American Thoracic Society/ERS recommendations [22]. 


\section{Analysis}

Data cleaning and reduction

As a first step in pre-processing, raw GC-MS files were converted into netCDF files; subsequent peak alignment was realised using XCMS [23]. After ComBat batch correction on the complete dataset of both the baseline and 12-18-month follow-up samples, GC-MS data was divided into training, replication and validation datasets [24]. Subsequently, datasets were normalised by adjusting the average and standard deviation of each individual GC-MS fragment to 0 and 1, respectively.

\section{Univariate analysis}

To select potentially relevant VOCs for the distinction between the drugs-detected versus no-drugs-detected groups, we applied a strategy reported by vAN OORT et al. [25]. This applies Wilcoxon sum-rank testing combined with computation of the area under the receiver operating characteristic curve (AUC) for each GC-MS fragment. Features with a p-value $<0.05$ and AUC $\geqslant 0.7$ (i.e. $\geqslant 70 \%$ ) were retained for further analysis. After filtering for multicollinearity through correlation analysis $(r \geqslant 0.95)$, spectra of GC-MS features retained after univariate analysis were provisionally identified based on National Institute of Standards and Technology library (version 2014) matching (https://chemdata.nist.gov). Compounds determined as instrument artefacts, i.e. silanes and siloxanes (Appendix C in [26]), or Tedlar bag-related substances [27] were considered as contaminants and removed for further analysis.

\section{Multivariate analysis}

A sparse partial least squares (SPLS) analysis was utilised on preserved GC-MS fragments in order to reveal the most optimal set of features for discrimination between groups of interest [28]. Optimal modelling settings for $\eta$ (thresholding parameter) and $K$ (number of hidden components) were objectively defined by the algorithms with 10-fold cross-validation. A composite score of the final set of selected variables was then calculated via a leave-one-out linear discriminant analysis. The diagnostic performance of the (breath-based) discriminant score versus urine LC-MS outcomes (drugs detected or no drugs detected) was tested by calculation of the AUC combined with $95 \%$ confidence interval.

\section{Validation}

Baseline outcomes were validated by rerunning the final two steps of the analysis, i.e. linear discriminant score and AUC calculation, on two datasets: follow-up samples of baseline patients ("replication") and an independent group of follow-up patients lacking paired (breath and urine) baseline samples ("validation").

\section{Correlation analysis}

Finally, the association between univariate preserved features and urinary concentrations of salbutamol, prednisone and prednisolone were determined on all samples (baseline and follow-up) together. After SPLS modelling, bootstrapped linear regression analysis (1000 replicates) was applied to test the robustness of the models.

All analyses were performed in RStudio version 1.0.136 (www.rstudio.com) using R version 3.3.3 (www. R-project.org) as engine, and supported by R packages: XCMS, data.table, MASS, pROC, caret, spls and sva.

\section{Results}

Complete data on both breath and urine were available for 48 baseline and 60 follow-up visits, occurring at eight research sites across five countries in Europe. Patient characteristics are summarised in table 1. A flowchart on patient enrolment is shown in figure 1.

There was no complete overlap between the patients from whom baseline and follow-up data were available. During the follow-up visits at 12-18 months, 30 patients were measured for the second time (called the "replication" set) and 30 patients for the first time (called the "validation" set). Of patients within the replication set, a few had a changed medication regime after the baseline visit. Four of them were withdrawn from OCS use, another four were prescribed an increased OCS dose and one patient was withdrawn from long-acting $\beta_{2}$-agonist use.

\section{Univariate analysis}

Analysis of the 48 baseline breath samples resulted in the detection of 7115 ion fragments. After pre-processing and combining outcomes of both the Wilcoxon sum-rank test $(\mathrm{p}<0.05)$ and AUC determination (AUC $\geqslant 70 \%$ ), the number of discriminatory features for GC-MS breathomics with regard to traces of salbutamol and OCS in urine (yes/no) by LC-MS were 79 and 28, respectively. Out of these 107 fragments, 48 were excluded either due to multicollinearity $(n=26)$, or were considered to represent either contaminants $(\mathrm{n}=13)$ or internal standards $(\mathrm{n}=9)$, which resulted in 49 "salbutamol" and 10 "OCS" related fragments. 


\begin{tabular}{|c|c|c|c|c|}
\hline & Training set & Replication set & Validation set & p-value \\
\hline Subjects $\mathrm{n}$ & 48 & 30 & 30 & \\
\hline Age years & $52.3 \pm 14.1$ & $54.9 \pm 11.9$ & $56.2 \pm 10.1$ & 0.38 \\
\hline Female & 52.1 & 50.0 & 60.0 & 0.71 \\
\hline Body mass index $\mathrm{kg} \cdot \mathrm{m}^{-2}$ & $30.5 \pm 5.8$ & $30.7 \pm 5.6$ & $28.7 \pm 6.3$ & 0.32 \\
\hline Smoking status & & & & 0.12 \\
\hline Current smoker & 2.1 & 3.3 & 16.7 & 0.12 \\
\hline Ex-smoker & 43.8 & 43.3 & 33.3 & 0.12 \\
\hline Nonsmoker & 54.2 & 53.3 & 50.0 & 0.12 \\
\hline Pack-years & $10.0(4.1-16.5)$ & $8.5(4.1-14.4)$ & $21.0(11.0-35.0)$ & 0.02 \\
\hline ICS(-LABA) prescription & 100 & 100 & 100 & NA \\
\hline OCS prescription" & 64.6 & 56.7 & 56.7 & 0.71 \\
\hline Total daily OCS dose & $10.0(9.4-15.0)$ & $10.0(7.5-15.0)$ & $10.0(5.0-20.0)$ & 0.95 \\
\hline SABA prescription ${ }^{+}$ & 56.3 & 53.3 & 56.7 & 0.96 \\
\hline LABA prescription ${ }^{+}$ & 37.5 & 40.0 & 33.3 & 0.86 \\
\hline Leukotriene modifier prescription ${ }^{+}$ & 47.9 & 36.7 & 40.0 & 0.59 \\
\hline Anti-IgE therapy prescription ${ }^{\S}$ & 20.8 & 23.3 & 16.7 & 0.81 \\
\hline Urinary prednisolone positive ${ }^{f}$ & 47.9 & 56.7 & 53.3 & 0.74 \\
\hline Urinary salbutamol positive ${ }^{f}$ & 29.2 & 43.3 & 20.0 & 0.14 \\
\hline Exacerbations per year & $1.00(1.00-2.25)$ & $1.50(0.00-2.75)$ & $1.00(0.00-2.75)$ & 0.60 \\
\hline Atopy positive $\# \#$ & 66.7 & 63.3 & 66.7 & 0.95 \\
\hline ACQ score & $1.86(1.43-3.00)$ & $1.64(1.29-2.86)$ & $2.00(1.18-3.18)$ & 0.85 \\
\hline Post-BD FEV $1 \%$ pred & $76.8 \pm 23.7$ & $80.8 \pm 21.6$ & $75.4 \pm 24.7$ & 0.65 \\
\hline Post-BD FEV $1 /$ FVC \% & $77.7 \pm 18.9$ & $76.0 \pm 15.6$ & $78.4 \pm 19.8$ & 0.87 \\
\hline$F_{\text {ENo }} \mathrm{ppb}$ & $33.5(20.0-53.0)$ & $37.8(19.6-55.3)$ & $18.5(13.5-27.5)$ & $<0.01$ \\
\hline Blood eosinophils $\times 10^{9} \mathrm{~L}^{-1}$ & $0.17(0.10-0.37)$ & $0.18(0.60-0.43)$ & $0.20(0.09-0.40)$ & 0.99 \\
\hline Blood neutrophils $\times 10^{9} \mathrm{~L}^{-1}$ & $5.31(4.08-8.01)$ & $6.27(4.79-7.67)$ & $6.24(4.85-7.61)$ & 0.60 \\
\hline
\end{tabular}

Data are presented as $\%$, mean \pm SD or median (interquartile range), unless otherwise stated. ICS: inhaled corticosteroid; LABA: long-acting $\beta_{2}$-agonist; NA: not applicable; OCS: oral corticosteroid; SABA: short-acting $\beta_{2}$-agonist; $A C Q$ : Asthma Control Questionnaire; BD: bronchodilator; FEV ${ }_{1}$ : forced expiratory volume in $1 \mathrm{~s} ; \%$ pred: \% predicted; FVC: forced vital capacity; $F_{\mathrm{ENO}}$ : exhaled nitric oxide fraction. \# : daily or alternate day prescription; " : normalised to prednisolone, among OCS users; ${ }^{+}$: daily prescription; ${ }^{\S}$ : weekly or monthly prescription; ${ }^{f}$ : traces in urine detected by liquid chromatography coupled with high-resolution mass spectrometry; ${ }^{\# \#}$ : presence of sensitisation on the skin prick test (wheal $\geqslant 3 \mathrm{~mm}$ ) or serum-specific $\lg E\left(\geqslant 0.35 \mathrm{kU} \cdot \mathrm{L}^{-1}\right)$. Differences between groups were tested using ANOVA (parametric) or Kruskal-Wallis (nonparametric) tests for continuous data and Pearson's Chi-squared test for categorical data.

\section{Multivariate analysis}

After SPLS modelling, seven discriminatory compounds remained for salbutamol and four for OCS (table 2).

The subsequent linear discriminant score calculation resulted in final baseline AUCs of $82.1 \%$ (95\% CI 70.4-93.9\%) for GC-MS breath versus LC-MS urine (salbutamol detected: yes/no) and 78.8\% (95\% CI 65.8-91.8\%) for GC-MS breath versus LC-MS urine (OCS detected: yes/no). These outcomes were replicated and validated using the two longitudinal datasets, resulting in outcomes for salbutamol of replication $67.0 \%$ (95\% CI 46.0-88.0\%) and validation 90.9\% (95\% CI 78.9-100.0\%) and for OCS of replication 70.1\% (95\% CI 50.8-89.5) and validation $82.1 \%$ (95\% CI 67.2-97.1\%) (table 2 and figures 2 and 3).

\section{Association with urinary levels of salbutamol and OCS}

SPLS modelling followed by regression analysis among classifying GC-MS features and urinary concentrations of salbutamol, prednisone and prednisolone resulted in three linear models with $\mathrm{R}^{2}$ of 0.10 (95\% CI $0.00-0.23$ ), 0.63 (95\% CI $0.33-0.94)$ and 0.29 (95\% CI $0.05-0.48$ ), respectively (all $\mathrm{p}<0.01$ ). The most stable model (association with prednisone) was based on four compounds: lysine (retention time $899.17 \mathrm{~s}$ ), glycolic acid (retention time $952.50 \mathrm{~s}$ ), 4-carene (retention time $1011.67 \mathrm{~s}$ ) and octanal (retention time 1046.48 s) (table 3 and figure 4).

\section{Discussion}

The present study examined the link between exhaled VOCs by GC-MS and traces of asthma medications in urine of severe asthma patients as measured by LC-MS. The distinction between the urine-based drugs-detected versus no-drugs-detected groups by the exhaled marker models resulted in moderate to good AUCs of $\sim 80 \%$ for both OCS and salbutamol. Both outcomes could be replicated (revisits) and 


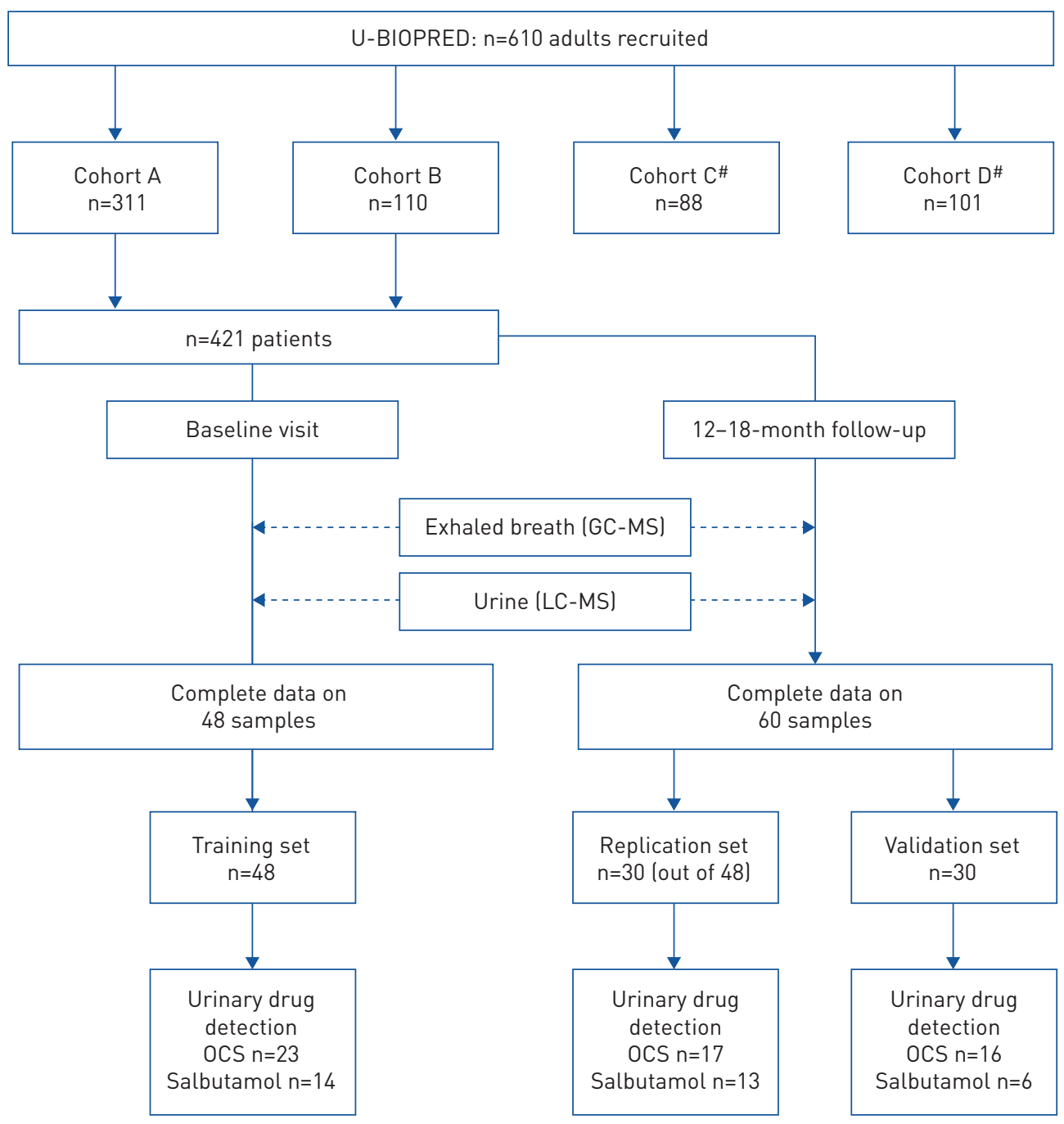

FIGURE 1 Flowchart of the patients enrolled in the study. GC: gas chromatography; MS: mass spectrometry; LC: liquid chromatography; OCS: oral corticosteroid. Cohort A: severe nonsmoking asthma (<5 pack-years). Cohort B: smokers and ex-smokers with severe asthma (>5 pack-years). Cohort C: mild/moderate nonsmoking asthmatic patients. Cohort D: healthy nonsmoking controls. Training set: all baseline samples of patients from cohorts A and B. Replication set: all available 12-18-month follow-up visit samples of patients included in the training set. Validation set: all available 12-18-month follow-up visit samples of patients without a baseline sample. \#: U-BIOPRED (Unbiased BIOmarkers in PREDiction of respiratory disease outcomes) cohorts $C$ and $D$ do not include severe asthma patients and did not have a 12-18-month follow-up.

validated (previously unsampled patients) using samples obtained during the 12-18-month follow-up visits. Our results indicate that profiles of exhaled VOCs might be useful for monitoring drug levels. These outcomes should be extended towards time series analysis, which argues for pharmacokinetics pathway and dose-ranging studies on asthma medication. Additionally, expanding to other types of asthma medication such as ICSs and long-acting $\beta_{2}$-agonists would strongly increase the added value of the proposed method of drug level monitoring.

To the best of our knowledge, this is the first study examining exhaled VOCs in relation to drug use as detected by urinary metabolites in asthma. The novelty of the present study is represented by linking two sources of excretion, i.e. urinary and exhaled VOCs, regarding the metabolism of asthma medication. Previously, breath profiles were linked to the metabolic effects of inhaled salbutamol [29] and eucalyptol [30] during a controlled drug regimen. The present study takes an additional step by relating exhaled metabolomics to urinary traces of the drugs.

There are a number of strengths of this study. First, both a longitudinal replication dataset and an independent validation cohort were available. Second, the analytical techniques for both urine and breath analysis were highly sophisticated and performed in experienced laboratories. Third, patient grouping 
TABLE 2 Gas chromatography-time-of-flight-mass spectrometry features adopted in the two distinct models (salbutamol detected: yes/no and oral corticosteroid (OCS) detected: yes/no) after sparse partial least squares (SPLS) modelling

\begin{tabular}{|c|c|c|c|}
\hline Compound name ${ }^{\#}$ & Fragment retention time $s$ & Salbutamol" & OCs $\pi$ \\
\hline Methyl-acetate & 498.90 & $\checkmark$ & \\
\hline 1-Propanol & 527.37 & $\checkmark$ & \\
\hline Butanal & 561.85 & $\checkmark$ & \\
\hline Methyl propionate & 611.23 & $\checkmark$ & \\
\hline 3-Methyl-butanal & 639.07 & $\checkmark$ & \\
\hline Chlorobenzene & 896.80 & & $\checkmark$ \\
\hline Butyrolactone & 929.10 & $\checkmark$ & \\
\hline Glycolic acid & 952.50 & & $\checkmark$ \\
\hline Octanal & 1046.48 & & $\checkmark$ \\
\hline Levomenthol & 1236.51 & & $\checkmark$ \\
\hline Carvone & 1301.60 & $\checkmark$ & \\
\hline
\end{tabular}
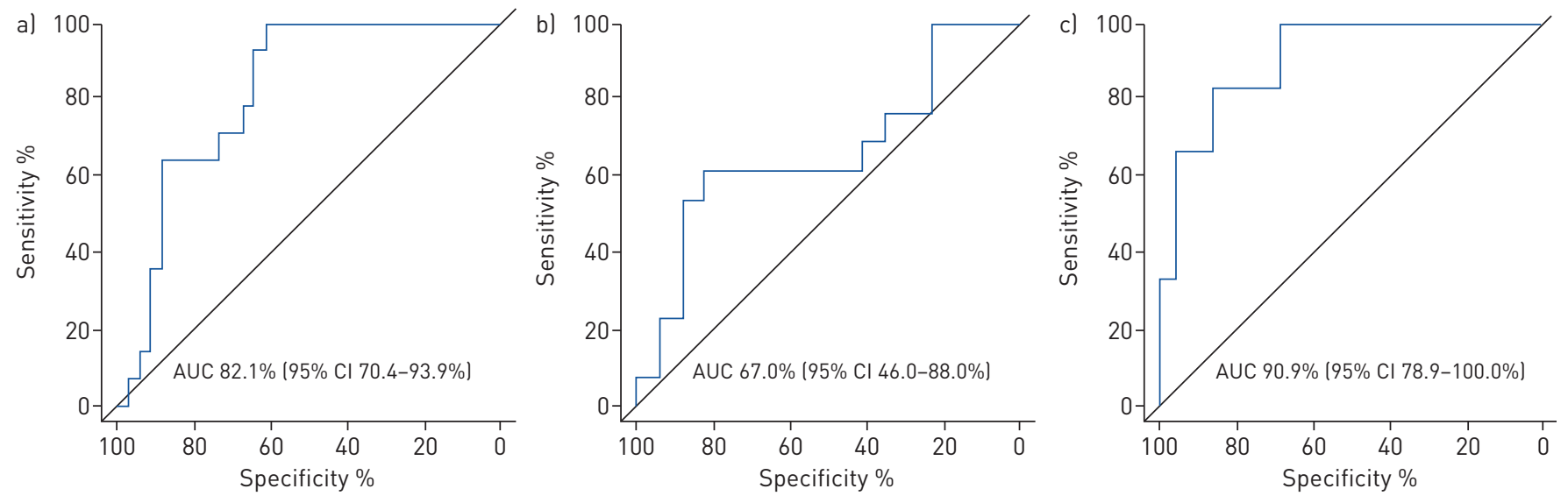

FIGURE 2 Receiver operating characteristic (ROC) curves for salbutamol: gas chromatography-mass spectrometry breath profiles versus liquid chromatography-mass spectrometry urine (salbutamol detected: yes/no) on a) baseline, b) replication and c) validation datasets. AUC: area under the ROC curve.
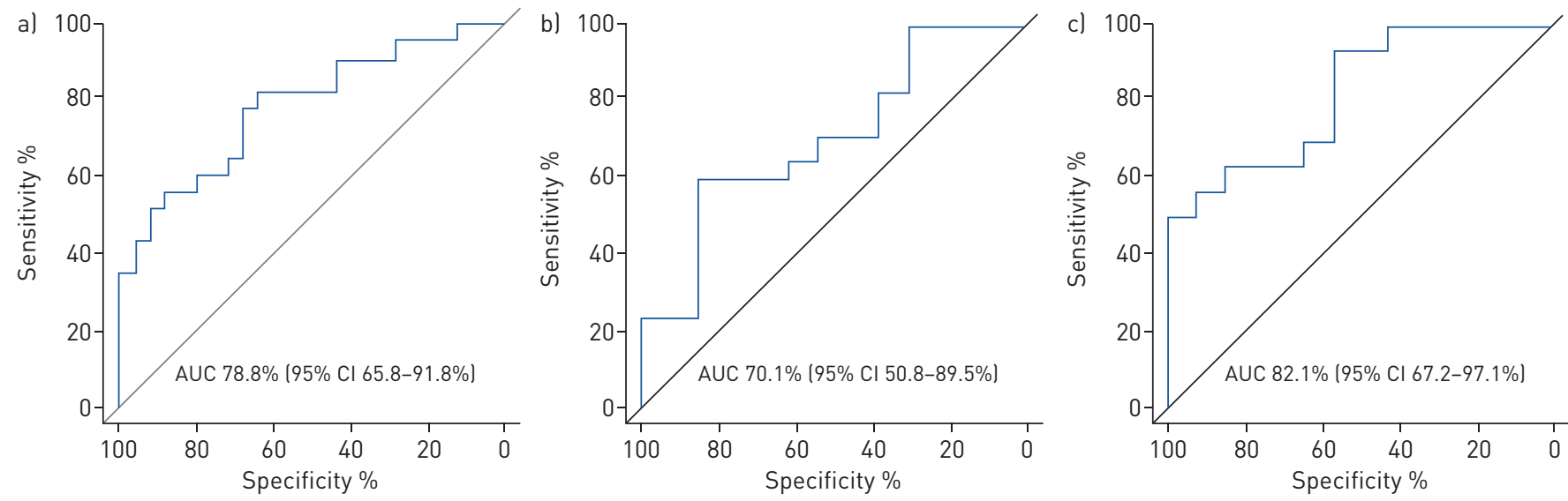

FIGURE 3 Receiver operating characteristic (ROC) curves for oral corticosteroid (OCS): gas chromatography-mass spectrometry breath profiles versus liquid chromatography-mass spectrometry urine (OCS detected: yes/no) on a) baseline, b) replication and c) validation datasets. AUC: area under the ROC curve. 
TABLE 3 Gas chromatography-time-of-flight-mass spectrometry features adopted in the sparse partial least squares-based model used for the bootstrapped linear regression analysis between exhaled markers and concentrations of prednisone in urine

Compound name ${ }^{\#}$

Fragment retention time $s$

$\begin{array}{lr}\text { Lysine } & 899.17 \\ \text { Glycolic acid } & 952.50 \\ \text { 4-Carene } & 1011.67 \\ \text { Octanal } & 1046.48\end{array}$

\#: most probable compound based on National Institute of Standards and Technology library matching.

regarding the use of asthma medications was based on detection of urinary metabolites by LC-MS, which might be more accurate than patient self-reporting or prescriptions [31]. Finally, this work arises from a pan-European study, with data originating from five different countries. Despite the multicentre and cross-border character of this study, which may have introduced variation within data, a link between urine and exhaled markers could be revealed. This may be a result of well-documented standardised operating protocols and the application of a thoroughly tested breath collection technique $[9,10]$.

We realise that this study has several limitations. First, due to the observational of this study, the specific time and dose of medication intake, and levels of hydration and nutrition, are uncertain. This is emphasised by the relative poor agreement between prescription of maintenance OCSs and the urinary prednisolone-positive patients within the training set. Additionally, there is a natural variability among individuals in the metabolism of the drugs $[4,5]$. Despite those limitations, a link between systemic markers of drug uptake in urine and exhaled markers was revealed that could be replicated and validated. Furthermore, since adherence within this study was based on a direct urinary measurement, the time of dosing is less relevant. A second limitation is related to the correlation analysis between urine and breath, which could only include patients with traces of medication in their urine. As a consequence, the number of samples was decreased compared with the first series of analyses of the study. This may have affected the robustness of the model, as represented by wide $95 \%$ confidence intervals. Therefore, the outcomes of our regression analysis should be interpreted with caution. Finally, the results obtained within the replication group are lower than those of the training and validation sets. Although we could not find a direct cause for this, we assume that a minor (technical) variation, exhaled breath or urine related, among a few patients within the relatively small group $(n=30)$ affected the results. The adequate outcomes of the validation set, which also builds upon the models of the training set, strengthen such an assumption.

How can we explain our findings? Out of 11 GC-MS features adopted in the two distinct models, two compounds are associated with downstream metabolic pathways. These are methyl-acetate for salbutamol and octanal for OCS, which are reported in the Kyoto Encyclopedia of Genes and Genomes database as a product of carbohydrate pyruvate metabolism and an aldehyde product of lipid peroxidation, respectively [32]. These outcomes fit earlier findings regarding changes in metabolic energy processes after intake of both drugs $[29,33,34]$. Regarding the compounds fostered in the presented regression analysis, lysine and

FIGURE 4 Associations between the exhaled marker-based sparse partial least squares (SPLS) model versus the log-transformed concentration of prednisone in urine by linear regression analysis $\left(\mathrm{R}^{2}=0.63,95 \%\right.$ Cl $0.33-0.94 ; p<0.01)(n=15)$

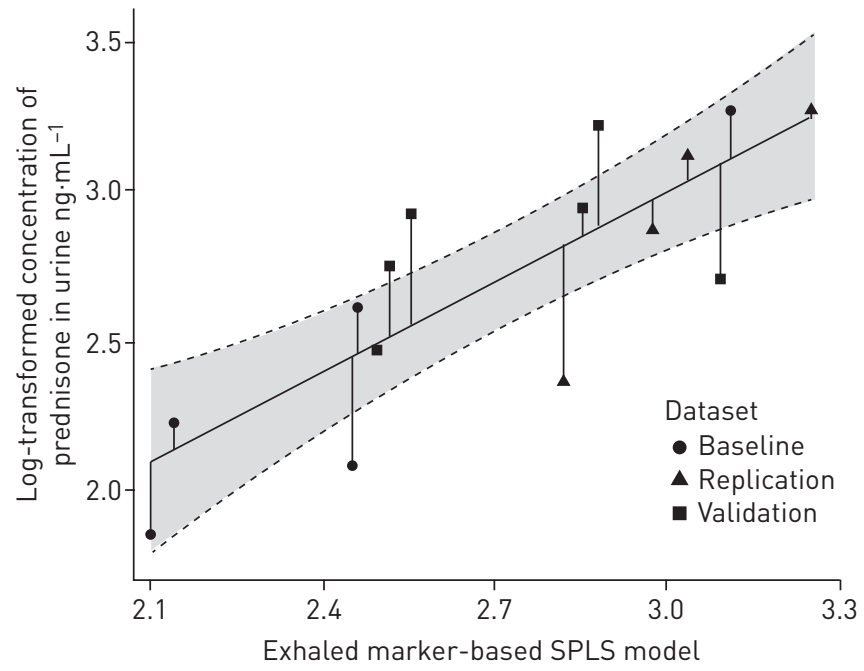


glycolic acid are of interest. Lysine, an essential amino acid, might have a link to the activation of genes by corticosteroids [35], while glycolic acid shows a remarkable similarity with the $\mathrm{COCH}_{2} \mathrm{OH}$ tail of prednisone. From the remaining set of compounds included in the final models, butyrolactone and 4-carene are described as potential biomarkers for lung and breast cancer, respectively [36, 37]; however, to the best of our knowledge, no direct link to asthma or medication use has yet been reported. Closely related to 4-carene in their chemical structure are 3-carene and 3-carane; interestingly, these two VOCs have been associated with lung infections [38]. Despite possible use as flavour additives (levomenthol and carvone) or a compound emitted by a microorganism (chlorobenzene) [39], no direct or indirect link was found for the other reported compounds.

What are the clinical implications of our data? The current study presents an association between excreted traces of asthma medication in urine and exhaled breath metabolomics. Therefore, breath might be a more favourable source to analyse biological responses and pharmacokinetics of asthma drugs than blood and urine, especially since it is noninvasive and practically inexhaustible. It suggests the advancement of current outcomes towards a breathomics-based, quick and noninvasive point-of-care tool for pharmacokinetics monitoring purposes or adherence testing, as are currently undertaken for the diagnosis of asthma, chronic obstructive pulmonary disease and lung cancer using gas sensor-driven eNose technology [11]. In addition, breathomics may qualify for rapid and frequent sampling of asthma drug use in sport science, e.g. testing misuse of drugs by athletes in real-time.

In conclusion, metabolomics of exhaled breath is associated with markers of recent drug use in urine. This suggests that breathomics qualifies for development into a point-of-care tool for monitoring drug level changes in both patients and athletes.

Acknowledgements: This paper is presented on behalf of the U-BIOPRED Study Group (see the supplementary material for details). The authors would like to thank the patients and their families for their participation.

Author contributions: P. Brinkman: study design, dry lab analysis and wrote the manuscript. W.M. Ahmed: dry lab analysis and amended the manuscript. C. Gómez: wet and dry lab analysis (LC-MS). H.H. Knobel: wet lab analysis (GC-MS). H. Weda: dry lab analysis (GC-MS). T.J. Vink and T.M. Nijsen: contributed to GC-MS analysis. C.E. Wheelock and S-E. Dahlén: contributed to LC-MS analysis. P. Montuschi: contributed to sample collection. R.G. Knowles: contributed to study design. S.J. Vijverberg and A.H. Maitland-van der Zee: amended the manuscript. P.J. Sterk: study design and amended the manuscript. S.J. Fowler: study design and wrote the manuscript.

Conflict of interest: P. Brinkman has nothing to disclose. W.M. Ahmed has nothing to disclose. C. Gómez has nothing to disclose. H.H. Knobel has a patent "Inline drying of breath sample" pending. H. Weda has nothing to disclose. T.J. Vink has nothing to disclose. T.M. Nijsen has nothing to disclose. C.E. Wheelock has nothing to disclose. S-E. Dahlén has participated in advisory boards for AstraZeneca, GSK, Merck, Novartis, Sanofi and Teva, outside the submitted work. P. Montuschi has nothing to disclose. R.G. Knowles is director of Knowles Consulting Ltd, outside the submitted work. S.J. Vijverberg has nothing to disclose. A.H. Maitland-van der Zee reports grants from Boehringer Ingelheim, personal fees for advisory board work from AstraZeneca, and personal fees for travel to meetings from Chiesi, outside the submitted work. P.J. Sterk reports grants (paid to institution) from Innovative Medicines Initiative covered by the European Union and the European Federation of Pharmaceutical Industries and Associations, during the conduct of the study; and reports a $4 \%$ interest and is a scientific advisor for Breathomix BV, outside the submitted work. S.J. Fowler reports personal fees for lectures and nonfinancial support to attend conferences from AstraZeneca, grants and personal fees for lectures from Boehringer Ingelheim, personal fees for lectures from Novartis, Teva and Chiesi, outside the submitted work.

Support statement: U-BIOPRED has received funding from the Innovative Medicines Initiative Joint Undertaking under grant agreement 115010, resources of which are composed of a financial contribution from the European Union's Seventh Framework Programme (FP7/2007-2013) and European Federation of Pharmaceutical Industries and Associations companies in-kind contribution (www.imi.europa.eu). Funding information for this article has been deposited with the Crossref Funder Registry.

\section{References}

1 Global Initiative for Asthma. Global Strategy for Asthma Management and Prevention. 2017. http://ginasthma.org/ 2017-gina-report-global-strategy-for-asthma-management-and-prevention Date last accessed: September 8, 2019.

2 Mokoka MC, Lombard L, MacHale EM, et al. In patients with severe uncontrolled asthma, does knowledge of adherence and inhaler technique using electronic monitoring improve clinical decision making? A protocol for a randomised controlled trial. BMJ Open 2017; 7: e015367.

3 Murphy AC, Proeschal A, Brightling CE, et al. The relationship between clinical outcomes and medication adherence in difficult-to-control asthma. Thorax 2012; 67: 751-753.

4 Fitch KD. The enigma of inhaled salbutamol and sport: unresolved after 45 years. Drug Test Anal 2017; 9: 977-982.

5 Zorgati H, Prieur F, Vergniaud T, et al. Ergogenic and metabolic effects of oral glucocorticoid intake during repeated bouts of high-intensity exercise. Steroids 2014; 86: 10-15.

6 World Anti Doping Agency. Medical Information to Support the Decisions of TUECs - Asthma. 2017. https:// www.wada-ama.org/en/resources/therapeutic-use-exemption-tue/medical-information-to-support-the-decisions-oftuecs-asthma Date last updated: January 17, 2019. Date last accessed: September 8, 2019. 
7 Ibrahim B, Basanta M, Cadden $\mathrm{P}$, et al. Non-invasive phenotyping using exhaled volatile organic compounds in asthma. Thorax 2011; 66: 804-809.

8 Fowler SJ, Basanta-Sanchez M, Xu Y, et al. Surveillance for lower airway pathogens in mechanically ventilated patients by metabolomic analysis of exhaled breath: a case-control study. Thorax 2015; 70: 320-325.

9 Dragonieri S, Schot R, Mertens BJ, et al. An electronic nose in the discrimination of patients with asthma and controls. J Allergy Clin Immunol 2007; 120: 856-862.

10 Hekking PP, Loza MJ, Pavlidis S, et al. Pathway discovery using transcriptomic profiles in adult-onset severe asthma. J Allergy Clin Immunol 2018; 141: 1280-1290.

11 de Vries R, Dagelet YWF, Spoor P, et al. Clinical and inflammatory phenotyping by breathomics in chronic airway diseases irrespective of the diagnostic label. Eur Respir J 2018; 51: 1701817.

12 King J, Mochalski P, Kupferthaler A, et al. Dynamic profiles of volatile organic compounds in exhaled breath as determined by a coupled PTR-MS/GC-MS study. Physiol Meas 2010; 31: 1169-1184.

13 Beauchamp J. Inhaled today, not gone tomorrow: pharmacokinetics and environmental exposure of volatiles in exhaled breath. J Breath Res 2011; 5: 037103.

14 Pleil JD. Breath biomarkers in toxicology. Arch Toxicol 2016; 90: 2669-2682.

15 Shaw DE, Sousa AR, Fowler SJ, et al. Clinical and inflammatory characteristics of the European U-BIOPRED adult severe asthma cohort. Eur Respir J 2015; 46: 1308-1321.

16 Bel EH, Sousa A, Fleming L, et al. Diagnosis and definition of severe refractory asthma: an international consensus statement from the Innovative Medicine Initiative (IMI). Thorax 2011; 66: 910-917.

17 van der Schee MP, Fens N, Brinkman P, et al. Effect of transportation and storage using sorbent tubes of exhaled breath samples on diagnostic accuracy of electronic nose analysis. J Breath Res 2013; 7: 016002.

18 Matabosch X, Pozo OJ, Pérez-Mañá C, et al. Identification of budesonide metabolites in human urine after oral administration. Anal Bioanal Chem 2012; 404: 325-340.

19 Matabosch X, Pozo OJ, Pérez-Mañá C, et al. Detection and characterization of prednisolone metabolites in human urine by LC-MS/MS. J Mass Spectrom 2015; 50: 633-642.

20 Mullen JE, Thorngren JO, Schulze JJ, et al. Urinary steroid profile in females - the impact of menstrual cycle and emergency contraceptives. Drug Test Anal 2017; 9: 1034-1042.

21 Miller MR, Hankinson J, Brusasco V, et al. Standardisation of spirometry. Eur Respir J 2005; 26: 319-338.

22 American Thoracic Society, European Respiratory Society. ATS/ERS recommendations for standardized procedures for the online and offline measurement of exhaled lower respiratory nitric oxide and nasal nitric oxide, 2005. Am J Respir Crit Care Med 2005; 171: 912-930.

23 Smith CA, Want EJ, O'Maille G, et al. XCMS: processing mass spectrometry data for metabolite profiling using nonlinear peak alignment, matching, and identification. Anal Chem 2006; 78: 779-787.

24 Johnson WE, Li C, Rabinovic A. Adjusting batch effects in microarray expression data using empirical Bayes methods. Biostatistics 2007; 8: 118-127.

25 van Oort PMP, de Bruin S, Weda H, et al. Exhaled breath metabolomics for the diagnosis of pneumonia in intubated and mechanically-ventilated intensive care unit (ICU)-patients. Int J Mol Sci 2017; 18: 449.

26 McMaster MC. GC/MS: A Practical Users Guide. New York, Wiley, 2007.

27 Beauchamp J, Herbig J, Gutmann R, et al. On the use of Tedlar bags for breath-gas sampling and analysis. J Breath Res 2008; 2: 046001.

28 Chung D, Keles S. Sparse partial least squares classification for high dimensional data. Stat Appl Genet Mol Biol 2010; 9: 1544-6115.

29 Gaugg MT, Engler A, Nussbaumer-Ochsner Y, et al. Metabolic effects of inhaled salbutamol determined by exhaled breath analysis. J Breath Res 2017; 11: 046004.

30 Beauchamp J, Kirsch F, Buettner A. Real-time breath gas analysis for pharmacokinetics: monitoring exhaled breath by on-line proton-transfer-reaction mass spectrometry after ingestion of eucalyptol-containing capsules. $J$ Breath Res 2010; 4: 026006.

31 Lim KG, Rank MA, Li JT, et al. How well does patient self-report predict asthma medication possession? Implications for medication reconciliation and adherence assessment $J$ Asthma 2010; 47: 878-882.

32 Kanehisa M, Furumichi M, Tanabe M, et al. KEGG: new perspectives on genomes, pathways, diseases and drugs. Nucleic Acids Res 2017; 45: D353-D361.

33 Fowler SJ, Lipworth BJ. Pharmacokinetics and systemic $\beta_{2}$-adrenoceptor-mediated responses to inhaled salbutamol. Br J Clin Pharmacol 2001; 51: 359-362.

34 Radhakutty A, Mangelsdorf BL, Drake SM, et al. Effects of prednisolone on energy and fat metabolism in patients with rheumatoid arthritis: tissue-specific insulin resistance with commonly used prednisolone doses. Clin Endocrinol 2016; 85: 741-747.

35 Barnes PJ, Adcock IM. How do corticosteroids work in asthma? Ann Intern Med 2003; 139: 359-370.

36 Ulanowska A, Kowalkowski T, Trawinska E, et al. The application of statistical methods using VOCs to identify patients with lung cancer. J Breath Res 2011; 5: 046008.

37 Silva CL, Passos M, Camara JS. Solid phase microextraction, mass spectrometry and metabolomic approaches for detection of potential urinary cancer biomarkers - a powerful strategy for breast cancer diagnosis. Talanta 2012; 89: 360-368.

38 Ahmed WM, Lawal O, Nijsen TM, et al. Exhaled volatile organic compounds of infection: a systematic review. ACS Infect Dis 2017; 3: 695-710.

39 Garbeva P, Hordijk C, Gerards S, et al. Volatiles produced by the mycophagous soil bacterium Collimonas. FEMS Microbiol Ecol 2014; 87: 639-649. 